\title{
Synovial sarcoma: current perspectives
}

This article was published in the following Dove Press journal:

Clinical Oncology in Adolescents and Young Adults

28 April 2016

Number of times this article has been viewed

\section{Bernadette Brennan}

Department of Paediatric Oncology, Royal Manchester Children's Hospital, Manchester, UK
Correspondence: Bernadette Brennan Royal Manchester Children's Hospital, Oxford Road, Manchester MI3 9WL, UK $\mathrm{Tel}+44$ I6I 7018430

$\mathrm{Fax}+441617018410$

Email Bernadette.brennan@cmft.nhs.uk
Abstract: Synovial sarcoma (SS) is a distinct soft tissue sarcoma, occurring across all ages, from young children to the elderly, but the incidence of SS peaks in young adults. Recently, its biology, specifically the biomarker genomic index, may prove to be the most important prognostic marker, explaining findings such as the positive effect of younger age on outcome. While believed to be a chemosensitive soft tissue sarcoma, surgery remains the most important modality of treatment for many people, especially in localized disease, plus or minus radiotherapy to improve local control. Moving forward, the demonstration that SS has multiple therapeutic targets such as vascular endothelial growth factor, and new emerging targets, allows us to start to consider different systemic therapeutic interventions other than just chemotherapy. This is particularly important for advanced/metastatic SS which, with conventional chemotherapy only, continues to have a very poor outcome. The way forward, therefore, is an all-age trial combining perhaps anti-angiogenesis agents with chemotherapy, focusing on those SS cases whose outcome, as determined by biology, site, or metastatic status, is poor with just conventional sarcoma chemotherapy. This also allows prospective evaluation of the role of genomic index and other biomarkers.

Keywords: children/adolescents, genomic index, targeted therapy, MAMS design

\section{Introduction}

After rhabdomyosarcoma, synovial sarcoma (SS) is the most common type of soft tissue sarcoma (STS) in childhood and adolescence, with a predilection for young adults. ${ }^{1}$ The incidence, however, in the context of STSs in adults is low, accounting for $<10 \%$ overall. $^{2}$ The incidence of SS may have changed over time due to more accurate diagnosis, with the recognition of a characteristic translocation involving chromosome 18 and $\mathrm{X}$, resulting in detection of one of the several types of fusion gene (SYT-SSX1, 2, and 4) in 90\% of cases. ${ }^{3,4}$ Surgery remains an important aspect of treatment for SS along with radiation, which may facilitate surgical resection. ${ }^{5}$ In pediatric and adolescent practice, less radiotherapy is used in view of the late effects. ${ }^{6}$ The role of age as a prognostic factor has been examined in single institutional studies, the US population-based Surveillance Epidemiology, the End Results (SEER) cancer registry, and the English national cancer registry. ${ }^{1,7}$ Ferrari et $\mathrm{al}^{8}$ demonstrated that children had a better outcome than adults, suggesting that this was probably due to the use of more chemotherapy in the younger age group. In adults, chemotherapy is not the standard of care, particularly in localized disease regardless of tumor size, but it is used in high-risk patients, ${ }^{8}$ or as part of investigational trials. ${ }^{9}$ However, the submit your manuscript $\mid$ www.dovepress.com Dovepress http://dx.doi.org/10.2147/COAYA.S91024
Clinical Oncology in Adolescents and Young Adults 2016:6 21-26

(c) (i) (5) 2016 Brennan. This work is published and licensed by Dove Medical Press Limited. The full terms of this license are available at https://www.dovepress.com/terms.php (c) ${ }_{\mathrm{BY}} \mathrm{NC}$ and incorporate the Creative Commons Attribution - Non Commercial (unported, v3.0) License (http://creativecommons.org/licenses/by-n/ 3.00 ). By accessing the work you hereby accept the Terms. Non-commercial uses of the work are permitted without any further permission from Dove Medical Press Limited, provided the work is properly attributed. For permission for commercial use of this work, please see paragraphs 4.2 and 5 of our Terms (https://www.dovepress.com/terms.php). 
better outcome in younger patients in part is probably explained by SS biology, in particular, the role of the genomic index (GI). ${ }^{10}$

Despite all the recent gains in knowledge, in particular, around biology, and a more defined role for chemotherapy, the outcome for SS has not significantly changed or, indeed, improved over recent years. Certainly, a recent report from the English national cancer registry on overall survival in the UK gives a 5-year relative survival of 57\%, which compares with other population-based series and has not significantly changed over the past 20-year period in the UK. ${ }^{11}$ The use of new agents, therefore, perhaps driven by a better understanding of the biology of STS, is badly needed. The number of STS in Phase I/II trials is small, due to their rarity, however there are novel therapies in trials worth pursuing in STS. The rarity means that much more collaborative studies are needed, in particular, collaborating within both pediatric and adult sarcoma groups - a truly all-age study. Traditionally, SS has been treated either within mixed STS adult trials or in specific trials but just in the pediatric age group: adolescents often sit between these two camps and therefore, do not always access trials. Rarity and small numbers may also force us to bring new agents earlier into Phase III studies, sometimes even generating hypotheses from data generated from Phase I trials.

\section{Epidemiology, biology, and predictors of outcome}

Two histologically distinct subtypes of SS can be distinguished, with each type having a strong association with the two main chimeric fusion genes, SYT-SSX1 and SYT-SSX2, from the specific translocation $\mathrm{t}(\mathrm{x} ; 18)(\mathrm{p} 11.2 ; \mathrm{q} 11.2)$ that occurs in $>95 \%$ of patients with SS. ${ }^{3,4}$ Biphasic tumors that contain both spindle- and epithelial-like cells tend to have the SYT-SSX1 fusion, whereas monophasic tumors that contain only spindle cells tend to have SYT-SSX2 fusion. ${ }^{12}$ The prognostic value of the pathology and/or type of translocation is conflicting. 2,12,13 This merely reflects the incompleteness of the series published with all the known prognostic values, including grade, stage, and size. Chromosomal instabilities detected by comparative genomic hybridization have been reported in SS. ${ }^{14}$ Lagarde et $\mathrm{al}^{15}$ developed this work further by establishing a 67-gene prognostic signature related to chromosome integrity, mitotic control, and genome complexity in sarcomas (Complexity Index in Sarcoma, CINSARC), and subsequently a genomic biomarker-GI - that is based on the number and type of chromosomal aberrations. The same group assessed GI in SS and has shown that a high GI score of 2 predicts for a very poor metastasis-free survival in both pediatric and adult SS. ${ }^{10}$ Indeed, the GI was higher in adults, indicating greater genomic instability, which offers a possible biological explanation for the poorer outcome in adults with SS. ${ }^{7,11,13}$ Age as a prognostic factor has been clearly shown in several cancer registry series. ${ }^{1,7,11,13}$ From the English national cancer registry data, the 5-year relative survival rate for the whole series across all ages from 1985 to 2008 was 56\% (95\% CI: 47\%-64\%). ${ }^{7,11}$ There were, however, significant differences $(P<0.05)$ in the 5-year relative survival rates between those patients aged $0-19$ years and those patients aged $\geq 20$ years, $72 \%$ (95\% CI: $46 \%-88 \%$ ) vs $53 \%(95 \%$ CI: $44 \%$; 62\%), respectively. ${ }^{7,11}$ The Dutch group was able to show that the outcome for the adolescent/young adult age group from their national registry cohort with localized SS falls between that of children and older adults, with a 5-year relative survival of $91 \%$ in children (aged $\leq 17$ years), $72 \%$ in adolescents/young adults (18-34 years), 58\% in adults (35-64 years), and 53\% in elderly ( $\geq 65$ years). ${ }^{13}$

Tumor size in SS is also predictive of outcome. Sultan et al, ${ }^{1}$ from the SEER cancer registry data of SS including all ages, demonstrated that tumors $>5 \mathrm{~cm}$ had a significantly worse outcome and the highest hazard ratio of 3.21 in predicting a poor outcome. The presence of metastases at diagnosis is clearly an important indicator of outcome, but the rate is the same regardless of the age of the patient. ${ }^{1}$ In our series from the English national cancer registry, the 5 -year relative survival was significantly lower at $7 \%$ for those with distant metastases compared to those without metastases at $65 \%(P \leq 0.001){ }^{7}$ Another prognostic factor to consider is site of tumor. The outcome for tumors at a nonextremity site compared with tumors of extremity is a 5-year relative survival of $34 \%$ vs $71 \%$, respectively. ${ }^{7}$ Again, like other clinical indicators in SS, the incidence of the primary tumor site does not differ across the age groups, trunk, and non-limb sites having the worst outcome. ${ }^{1,7}$ Poor outcome in SS arising in non-limb sites has also been demonstrated in a smaller Italian series. ${ }^{16}$

A novel and yet to be fully explored prognostic indicator is the role of socioeconomic deprivation, using the income domain of the Index of Multiple Deprivation 2010 (ID), a specific measure available in the UK. ${ }^{17}$ Socioeconomic status has been shown to be a significant predictor of outcome in lung, colon, and breast cancer with those patients with lower socioeconomic status having a worse outcome. ${ }^{18-20}$ In the study of all stages of SS from the English national cancer registry, patients with the highest ID score and hence the worst deprivation had the worst outcome, but this was 
due to the adult cohort in the series, as a high ID score was not significant in the younger age group ( $0-18$ years).$^{7}$ This may be plausible due to diagnostic delays, either professional or patient, or poor awareness of this tumor, but no data on time to diagnosis or the diagnostic pathway were available to explore this further in the English national cancer registry. The prognostic role of deprivation on the survival of SS has not been studied previously, but in Ewing's sarcomas registered in the Californian-population-based cancer registry, survival was significantly lower in the most deprived groups. $^{21}$

\section{Role of chemotherapy}

For the majority of SSs, especially those which are localized and operable, surgery plus or minus radiotherapy remain the standard of care. In the SEER series from 1983 to 2005, of the 1,268 patients with all stages of SS, fewer than $10 \%$ had no local treatment, including surgery, regardless of patient age, but $50 \%$ of the whole series received radiotherapy. ${ }^{1}$ In a more contemporary series of patients with non-metastatic extremity SS only, from the US National Cancer Database (2000-2009), 32\% had some form of radiotherapy, either pre- or postoperatively, but only $12 \%$ had some form of chemotherapy. ${ }^{22}$ These differences between the series on therapy modalities merely reflect the different stages of SS included in each series, as the series from the US national cancer database only included localized extremity SS. ${ }^{22}$ In the recent analysis from the Dutch cancer registry, of 461 localized SS patients, nearly 50\% received radiotherapy, but $<1 \%$ had chemotherapy and $34 \%$ had surgery only. ${ }^{13}$

In the pediatric/adolescent oncology world, it has often been believed that the better outcomes in these age groups children and adolescents - compared to that in adults, are due to the more frequent use of chemotherapy in children. ${ }^{23}$ Chemotherapy usage in adults has often been reserved for large, stage III patients or those with distant metastases, hence selecting those SS expected to have a worse outcome. ${ }^{1,2}$ Two approximately contemporary Italian hospital-based series have contrasting results. At Milan's Instituto Nazionali dei Tumori, among 255 patients with localized disease more chemotherapy was given to younger patients, suggesting that this accounted for their better outcome. ${ }^{8}$ At Bologna's Instituto Ortopedico Rizzoli, however, among patients with localized disease there was no difference in chemotherapy usage between age groups, but children had a better survival than adults. ${ }^{24}$ The lack of treatment effect, in particular, chemotherapy, has been confirmed in the Dutch cancer registry study in localized $\mathrm{SS}$, which also demonstrated that younger age is again important for a better outcome, regardless of site or tumor size or use of chemotherapy. ${ }^{13}$

Perhaps what we are now clearer about is those patients in whom we may avoid adjuvant chemotherapy, ie, patients with good risk, localized disease. In our series of $71 \mathrm{UK}$ pediatric and adolescent patients with SS, we defined a population of 21 patients who received surgery only with an excellent outcome. They had small tumors $(<5 \mathrm{~cm})$ with an $\mathrm{R}_{0}$ resection. ${ }^{25}$ In the recent European Pediatric Soft tissue Sarcoma Study Group, pediatric and adolescent prospective series of 138 patients with localized SS enrolled on the study protocol between 2005 and 2012, the outcome for 24 low-risk patients (tumor $\leq 5 \mathrm{~cm}$ in size, completely resected, at a limb site) was excellent with surgery only, with only two local relapses, who were subsequently rescued with further local treatment and remain in a complete response. ${ }^{23}$ Therefore, in both Europe and USA, it is now standard to not receive chemotherapy, regardless of age, in small resected SSs.

In adult STS randomized studies, an assessment of the role of adjuvant chemotherapy for all STSs has failed to show a benefit for adjuvant chemotherapy, hence the European Society for Medical Oncology (ESMO)/European Sarcoma Network Working Group guidelines for STS has recommended that adjuvant chemotherapy for localized SS is only indicated within the context of a randomized clinical trial. ${ }^{26}$ These studies, however, include all types of STS, which may dampen the effect of chemotherapy in SS, which is believed to be the most chemosensitive of the non-rhabdomyosarcoma STSs. ${ }^{23}$ Certainly, retrospective studies in SS treated with neoadjuvant/adjuvant chemotherapy give contrasting results, in particular, for the use of ifosfamide-based chemotherapy: some show improved survival compared to patients treated with surgery only, ${ }^{27}$ but in contrast, Italiano et $\mathrm{al}^{28}$ could not demonstrate any benefit from neoadjuvant chemotherapy in a French series. Both studies were non-randomized, however.

The chemotherapy regimen chosen for advanced SS is either ifosfamide in combination with doxorubicin, or doxorubicin alone, but no type or combination seems to be advantageous. ${ }^{29}$ Again, the literature in this area is not quite so clear, as the randomized studies included all STS, whereas studies in SS are usually retrospective and demonstrate a more positive outcome and better response to chemotherapy. ${ }^{29-31}$ In the pooled analysis of the European Organization for Research and Treatment of Cancer (EORTC) studies for advanced STS, the SS subgroup showed a better response rate of 33\% with ifosfamide-based regimens, compared to $25 \%$ with doxorubicin. ${ }^{31}$ Of the more recent "new" chemotherapies in sarcoma, trabectedin has shown some promise 
in SS. ${ }^{32}$ Its role at present, however, is still in the context of advanced and metastatic disease where other chemotherapy regimens have failed. ${ }^{33}$

Clearly, the way forward is to propose a randomized study for SS alone, looking at all the "standard" chemotherapy regimens, perhaps "picking the winner" by means of a multi-arm multistage (MAMS) design, to take forward a standard chemotherapy backbone, to which to add newer drugs or indeed other arms as they become available. ${ }^{34}$ This kind of design allows "newer" chemotherapy drugs such as trabectedin to be added to the randomization, which has shown promising response rates in SS either on its own or in combination. ${ }^{35}$ The MAMS design also allows us to add new targeted agents as they come into clinical trials, especially those to specific targets in SS. Perhaps for a rare tumor such as SS, with small numbers available to enter randomized studies, the major advantage for an MAMS design is the ability to answer the question with small numbers of subjects in a much more efficient way.

\section{New targeted treatments}

In STS, there is evidence of angiogenesis playing a critical role in tumor progression, and hence anti-angiogenic agents seem to be targeted agents worth considering. This can either be with multiple tyrosine kinase inhibitors (TKIs) such as vascular endothelial growth factor receptor inhibitors, sorafenib, pazopanib, sunitinib, and cediranib, or with monoclonal antibodies such as bevacizumab. ${ }^{36}$ Furthermore, the overexpression of platelet-derived growth factor receptor antigen in SS, which is also targeted by these TKIs, makes vascular endothelial growth factor inhibitors most likely to have a positive outcome. ${ }^{37}$

Probably, the most suitable candidates to consider as TKI for SS are sorafenib and pazopanib. ${ }^{36,38}$ For sorafenib, singleagent Phase II studies in STSs have shown responses in SS, and at least comparable findings to conventional chemotherapy in advanced or previously treated SSs. ${ }^{39-41}$ Of possibly more potential is the combination with ifosfamide, in which the Spanish sarcoma group has done in a Phase I study, as it is unlikely that TKIs have a role on their own, but maybe in enhancing the effect of chemotherapy ${ }^{38}$ The Spanish sarcoma group is currently performing a Phase II study in the same SS cohort. For pazopanib, there are more data in STS to support its future use in SS trials, hence its approval in the USA for STS in relapsed patients following conventional chemotherapy. The PALLETTE study demonstrated its benefit in STS vs a placebo, including responses in $\mathrm{SS} .{ }^{42}$ Again, studies are ongoing in limb STS prior to chemotherapy and surgery.
Another possible target is the insulin growth factor (IGF) system, specifically IGF-1, with the availability of IGF-1 receptor inhibitors. Several trials in sarcoma, including SS, have explored IGF-1 receptor inhibitors, demonstrating both partial response and stable disease ${ }^{43}$ Again, its benefit in combination with chemotherapy in sarcomas is being further explored, and this will include SS cases.

As there is a high expression of cancer/testis (CT) antigen in SS, specifically NY-ESO-1, which is highly immunogenic, the role of genetically engineered targeted T-lymphocytes to this antigen has been explored in SS. ${ }^{44,45}$ Ipilimumab, a monoclonal antibody that enhances immunity against $\mathrm{CT}$ antigen NY-ESO-1, is again a possible immunotherapy in SS, but a study of the antibody in SS closed early due to lack of activity and recruitment despite a theoretical therapeutic advantage. ${ }^{46}$ The role of immunotherapy, in particular in combination with chemotherapy, certainly needs further exploration as it might be a more efficient way of presenting the antigen in SS.

\section{Conclusion}

For SS, the emerging evidence of the different biological subtypes as defined by GI, previously reflected in the better outcomes in younger patients, should allow us to define the different populations of patients with SS who warrant different approaches to their treatment. There is a proviso here, as the role of GI needs defining prospectively in an "all-age" study, alongside therapeutic interventions.

For those with good biology, as defined by a low GI and, usually, small localized tumors, then local control either with surgery alone in small tumors at good prognostic sites may be enough. Radiation, as well, is usually required for those with larger tumors. For those with localized "poor" risk tumors, as defined by biology or site, and for those with metastatic tumors, further therapeutic intervention needs to be considered alongside conventional "sarcoma chemotherapy". More mature data now on anti-angiogenic agents such as pazopanib or sorafenib may be the first step, and particularly as we may have data from other sarcomas on their use in combination with chemotherapy, plus they are commercially available. The role of immunotherapy in SS, particularly against the CT NY-ESO-1 antigen, is of particular interest, but will be difficult to deliver in all clinical settings due to the inability to generate genetically engineered autologous T-cells.

The rarity of SS is often given as a reason not to develop specific trials in SS alone, but using innovative statistical designs; perhaps with one of the available targeted agents in a multiple arm randomized study, then this may be possible. In particular, it should be possible to consider a randomized 
study across all ages involving multiple countries and sites, focusing on those SS cases whose outcome, as determined by biology, site, or metastatic status, is poor with just conventional sarcoma chemotherapy.

\section{Disclosure}

The author reports no conflicts of interest in this work.

\section{References}

1. Sultan I, Rodriguez-Galindo C, Saab R, Yasir S, Casanova M, Ferrari A. Comparing children and adults with synovial sarcoma in the surveillance, epidemiology, and end results program, 1983 to 2005: an analysis of 1268 patients. Cancer. 2009;115:3537-3547.

2. Corey RM, Swett K, Ward WG. Epidemiology and survivorship of soft tissue sarcomas in adults: a national cancer database report. Cancer Med. 2014;3:1404-1415.

3. dos Santos NR, de Brujin DR, Geurts van Kessel A. Molecular mechanisms underlying human synovial sarcoma development. Genes Chromosomes Cancer. 2001;30:1-14.

4. Ladanyi M. Fusion of the SYT and SSX genes in synovial sarcoma. Oncogene. 2001;20:5755-5762.

5. Pollack A, Zagars GK, Goswitz MS, Pollock RA, Feig BW, Pisters PW. Preoperative vs. postoperative radiotherapy in the treatment of soft tissue sarcomas: a matter of presentation. Int J Radiat Oncol Biol Phys. 1998;42:563-572.

6. Brecht IB, Ferrari A, Int-Veen C, et al. Grossly-resected synovial sarcoma treated by the German and Italian pediatric soft tissue sarcoma cooperative groups: discussion on the role of adjuvant therapies. Pediatr Blood Cancer. 2006;46:11-17.

7. Brennan B, Dennis N, Broggio J, Francis M. Outcome and the effect of age and socioeconomic status in 1318 patients with synovial sarcoma in the English National Cancer Registry: 1985 - 2009. Abstract paper 023 presented at: CTOS; October 17, 2014; Berlin.

8. Ferrari A, Gronchi A, Casanova M, et al. Synovial sarcoma: a retrospective analysis of 271 patients of all ages treated at a single institution. Cancer. 2004;101:627-634.

9. Pervaiz N, Colterjohn N, Farrokhyar F, Tozer R, Figueredo A, Ghert M. A systematic meta-analysis of randomized controlled trials of adjuvant chemotherapy for localized resectable soft-tissue sarcoma. Cancer. 2008;3:573-581.

10. Lagarde P, Przybyl J, Brulard C, et al. Chromosome instability accounts for reverse metastatic outcomes of pediatric and adult synovial sarcomas. J Clin Oncol. 2013;31:608-615.

11. Dennis N, Francis M, Lawrence G. Soft Tissue Sarcoma Incidence and Survival: Tumours Diagnosed in England between 1985 and 2009; London: National Cancer Intelligence Network; NCIN report R12/06; 2012.

12. Antonescu CR, Kawai A, Leung DH, et al. Strong association of SYT-SSX fusion type and morphologic epithelial differentiation in synovial sarcoma. Diagn Mol Pathol. 2000;9:1-8.

13. Vlenterie M, Ho VK, Kaal SE, Vlenterie R, Haas R, van der GraafWT. Age as an independent prognostic factor for survival of localised synovial sarcoma patients. Br J Cancer. 2015;113:1602-1606.

14. Nakagawa $Y$, Numoto $\mathrm{K}$, Yoshida A, et al. Chromosomal and genetic imbalances in synovial sarcoma detected by conventional and microarray comparative genomic hybridization. $J$ Cancer Res Clin Oncol. 2006;132:444-450.

15. Lagarde P, Pérot G, Kauffmann A, et al. Mitotic checkpoints and chromosome instability are strong predictors of clinical outcome in gastrointestinal stromal tumors. Clin Cancer Res. 2012;18:826-838.

16. Ferrari A, Bisogno G, Alaggio G, et al. Synovial sarcoma of children and adolescents: the prognostic role of axial sites. Eur J Cancer. 2008;44:1202-1209.
17. Gov.UK [webpage on the Internet]. Communities and Local Government: Indices of Deprivation. Available from: http://www.communities. gov.uk/corporate/researchandstatistics/statistics/subject/indicesdeprivation. Accessed March 18, 2010.

18. Riaz SP, Horton M, Kang J, Mak V, Lüchtenborg M, Møller H. Lung cancer incidence and survival in England: an analysis by socioeconomic deprivation and urbanization. J Thorac Oncol. 2011;6: 2005-2010.

19. Bharathan B, Welfare M, Borowski DW, et al. Impact of deprivation on short- and long-term outcomes after colorectal cancer surgery. $\mathrm{Br} J$ Surg. 2011;98:854-865.

20. Downing A, Prakash K, Gilthorpe MS, Mikeljevic JS, Forman D. Socioeconomic background in relation to stage at diagnosis, treatment and survival in women with breast cancer. Br J Cancer. 2007;96(5): 836-840.

21. Lee J, Hoang BH, Ziogas A, Zell JA. Analysis of prognostic factors in Ewing sarcoma using a population-based cancer registry. Cancer. 2010;116:1964-1973.

22. Sherman KL, Wayne JD, Chung J, et al. Assessment of multimodality therapy use for extremity sarcoma in the United States. J Surg Oncol. 2014;109:395-404.

23. Ferrari A, De Salvo GL, Brennan B, et al. Synovial sarcoma in children and adolescents: the European Pediatric Soft tissue Sarcoma Study Group prospective trial (EpSSG NRSTS 2005). Ann Oncol. 2014;26(3): 567-572.

24. Palmerini E, Staals EL, Alberghini M, et al. Synovial sarcoma: retrospective analysis of 250 patients treated at a single institution. Cancer. 2009;115:2988-2998.

25. Brennan B, Stevens M, Kelsey A, Stiller CA. Synovial sarcoma in childhood and adolescence: a retrospective series of 77 patients registered by the Children's Cancer and Leukaemia Group between 1991 and 2006. Pediatr Blood Cancer. 2010;55:85-90.

26. The ESMO/European Sarcoma Network Working Group. Soft tissue and visceral sarcomas: ESMO clinical practice guidelines for diagnosis, treatment and follow-up. Ann Oncol. 2014;25(suppl 3): iii102-iii112.

27. Eilber FC, Brennan MF, Eilber FR, et al. Chemotherapy is associated with improved survival in adult patients with primary extremity synovial sarcoma. Ann Surg. 2007;246:105-113.

28. Italiano A, Penel N, Robin YM, et al. Neo/adjuvant chemotherapy does not improve outcome in resected primary synovial sarcoma: a study of the French Sarcoma Group. Ann Oncol. 2009;20:425-430.

29. Judson I, Verweij J, Gelderblom H, et al. Doxorubicin alone versus intensified doxorubicin plus ifosfamide for first-line treatment of advanced or metastatic soft-tissue sarcoma: a randomised controlled phase 3 trial. Lancet Oncol. 2014;15:415-423.

30. van der Graaf WT, Elisa R, Gronchi A, et al; EORTC Soft Tissue and Bone Sarcoma Group. Outcome of chemotherapy in synovial sarcoma patients: review of 15 clinical trials from EORTC involving advanced SyS compared to other soft tissue sarcoma. Abstract paper 024 presented at: CTOS; October 17, 2014; Berlin.

31. Sleijfer S, Ouali M, van Glabbeke M, et al. Prognostic and predictive factors for outcome to first-line ifosfamide-containing chemotherapy for adult patients with advanced soft tissue sarcomas: an exploratory, retrospective analysis on large series from the European Organization for Research and Treatment of Cancer-Soft Tissue and Bone Sarcoma Group (EORTC-STBSG). Eur J Cancer. 2010;46:72-83.

32. Le Cesne A, Cresta S, Maki RG, et al. A retrospective analysis of antitumour activity with trabectedin in translocation-related sarcomas. Eur J Cancer. 2012;48:3036-3044.

33. Sanfilippo R, Dileo P, Blay JY, et al. Trabectedin in advanced synovial sarcomas: a multicenter retrospective study from four European institutions and the Italian Rare Cancer Network. Anticancer Drugs. 2015;26(6):678-681.

34. Jaki T, Hampson LV. Designing multi-arm multi-stage clinical trials using a risk-benefit criterion for treatment selection. Stat Med. 2016; 35(4):522-533. 
35. Bui-Nguyen B, Butrynski JE, Penel N, et al. A phase IIb multicentre study comparing the efficacy of trabectedin to doxorubicin in patients with advanced or metastatic untreated soft tissue sarcoma: the TRUSTS trial; European Organisation for Research and Treatment of Cancer Soft Tissue and Bone Sarcoma Group (EORTC/STBSG) and the Sarcoma Alliance for Research through Collaboration (SARC). Eur J Cancer. 2015;51:1312-1320.

36. Chao C, Al-Saleem T, Brooks JJ, Rogatko A, Kraybill WG, Eisenberg B. Vascular endothelial growth factor and soft tissue sarcomas: tumor expression correlates with grade. Ann Surg Oncol. 2001;8(3):260-267.

37. Ho AL, Vasudeva SD, Laé M, et al. PDGF receptor alpha is an alternative mediator of rapamycin-induced Akt activation: implications for combination targeted therapy of synovial sarcoma. Cancer Res. 2012;72(17):4515-4525.

38. Martín-Liberal J, López-Pousa A, Broto JM, et al. Phase I trial of sorafenib in combination with ifosfamide in patients with advanced sarcoma: a Spanish group for research on sarcomas (GEIS) study. Invest New Drugs. 2014;32(2):287-294.

39. Maki RG, D’Adamo DR, Keohan ML, et al. Phase II study of sorafenib in patients with metastatic or recurrent sarcomas. J Clin Oncol. 2009;27(19):3133-3140.

40. Basso U, Brunello A, Bertuzzi A, Santoro A. Sorafenib is active on lung metastases from synovial sarcoma. Ann Oncol. 2009;20(2):386-387.
41. Santoro A, Comandone A, Basso U, et al. A. Phase II prospective study with sorafenib in advanced soft tissue sarcomas after anthracyclinebased therapy. Ann Oncol. 2013;24(4):1093-1098.

42. van der Graaf WT, Blay JY, Chawla SP, et al. Pazopanib for metastatic soft-tissue sarcoma (PALETTE): a randomised, double-blind, placebocontrolled phase 3 trial. Lancet. 2012;379:1879-1886.

43. Schöffski P, Adkins D, Blay JY, et al. An open-label, phase 2 study evaluating the efficacy and safety of the anti-IGF-1R antibody cixutumumab in patients with previously treated advanced or metastatic soft-tissue sarcoma or Ewing family of tumours. Eur $J$ Cancer. 2013;49(15):3219-3228.

44. Lai JP, Robbins PF, Raffeld M, et al. NY-ESO-1 expression in synovial sarcoma and other mesenchymal tumors: significance for NY-ESO-1-based targeted therapy and differential diagnosis. Mod Pathol. 2012;25(6):854-858.

45. Robbins PF, Morgan RA, Feldman SA, et al. Tumor regression in patients with metastatic synovial sarcoma and melanoma using genetically engineered lymphocytes reactive with NY-ESO-1. J Clin Oncol. 2011;29(7):917-924.

46. Maki RG, Jungbluth AA, Gnjatic S, et al. A pilot study of anti-CTLA4 antibody ipilimumab in patients with synovial sarcoma. Sarcoma. 2013;2013:168145.
Clinical Oncology in Adolescents and Young Adults

\section{Publish your work in this journal}

Clinical Oncology in Adolescents and Young Adults is an international, peer-reviewed, open access journal publishing original research, reports, editorials, reviews and commentaries on all aspects of epidemiology, diagnosis and treatment of cancers in adolescents and young adults. The manuscript management system is completely

\section{Dovepress}

online and includes a very quick and fair peer-review system. Visit http://www.dovepress.com/testimonials.php to read real quotes from published authors.

\footnotetext{
Submit your manuscript here: http://www.dovepress.com/clinical-oncology-in-adolescents-and-young-adults-journal
} 